\title{
Hearing results after ossiculoplasty with incus transposition, cortical bone autografting and bone cement
}

Original Article

\author{
Essam Abd El Wanees Beharey, Mohammed Qamar El Sharnouby, Ahmad \\ Helmy Zayan, Ashraf Ali El-Demerdash \\ Department of Otolaryngology, Faculty of Medicine, Menoufia University, Menoufia, Egypt
}

\begin{abstract}
Background: This study assesses hearing results after ossiculoplasty with incus transposition (IT), cortical bone autograft (CBA) and bone cement (BC).

Methods: A total of 21 patients with mild/moderate conductive hearing loss were enrolled in our study. Patients were assigned to each of the three techniques according to length of incus defect. Pre-operative and 6 months post-operative audiograms (PTA) were obtained and results of air bone gap (ABG) were statistically analyzed.

Results: A total of 7 patients were included in each technique. BC ABG was decreased from 26.57 (5.06) (mean (SD)) to 10.86 (6.34) with mean gain 15.71 (5.85) For IT group, the mean for pre- ABG was 33.57 with SD 5.13, it became 15.86 (1.86) after 6 months of follow-up with 17.71 (3.40) gain. For CBA, pre- ABG mean was 35.86 (5.67) that finally became 19.14 (2.41) achieving ABG gain 16.71 (6.68). No significant statistical difference between pre-post gain in all three surgical procedures ( $\mathrm{p}$-value $=0.80)$. Regarding side effects in each technique, infections was noted in one case of IT and two cases of CBA, transient infection was found in one case of IT and CBA, and transient vertigo in one case of $\mathrm{BC}$ with no significant difference in adverse effects between the three group ( $\mathrm{P}$-value $=0.494$ ).

Conclusions: Both BC, IT and CBA are reliable techniques for ossiculoplasty with good postoperative hearing results. The choice of the proper technique depends on length of incus defects.
\end{abstract}

Key Words: Bone cement, cortical bone autograft, incus transposition, ossiculoplasty.

Received: $26^{\text {th }}$ July 2018, Accepted: $14^{\text {th }}$ August 2019

Corresponding Author: Ahmad Helmy Zayan, MSc, Department of Otolaryngology, Faculty of Medicine, Menoufia University, Menoufia, Egypt, Tel.: +20 1092992202, E-mail: ahmad_zayan@yahoo.com.

ISSN: 2090-0740, March 2020 Vol. 21, No. 1.

\section{INTRODUCTION}

Ossicular erosions or destruction as a consequence of chronic suppurative otitis media are relatively common. It usually presents with erosions of the long process of incus due to its position and the course of blood supply. Consequently, it results in mild to moderate conductive hearing loss. Ossiculoplasty remains one of the challenging operations for otology surgeons. From the mid-1990s till our current time, various surgical techniques and materials were proposed for this purpose. Autografting, gold prosthesis, and hydroxyapatite are among the available materials. However, there is no solid literature agreement on the best available material or technique ${ }^{[1]}$.

As stated before, the long process of the incus is a quite common site for ossicular discontinuity leading to the incudo-stapedial joint separation. It presents around 60 percent of ossicular defects with intact malleus handle and intact mobile stapes. As a consequence, type II or III tympanoplasty is considered the appropriate procedure according to Wullstein classification ${ }^{[2]}$. One of the nowadays popular techniques for the incudo-stapedial bridging is bone cement (BC). In a recent study conducted by Baylancicek et al 2014 $4^{[3]}$, BC was found to be a reliable, easily available technique with results of hearing gain comparable to partial ossicular replacement prosthesis
(PORP) with no significant statistical difference between both procedures. As well, Bartel et al ${ }^{[4]}$ conducted a metaanalysis in 2018 and concluded that incus transposition (IT) gave comparable results to PORP especially regarding decibels gain. In the presence of difficulties in IT, cortical bone autograft (CBA) might present a good substitute. In our study, we aim to present the hearing results after ossiculoplasty with IT, CBA and BC.

\section{METHODS}

Our study was carried on patients in the Department of Otolaryngology, Head and Neck Surgery at Menoufia University Hospital, Menoufia, Egypt. Approval from Institutional Review Board of Menoufia University and informed patient consents in March 2018, were obtained before patient recruitment.

\section{Inclusion and exclusion criteria}

All patients with a moderate degree of conductive hearing loss resulting from either chronic suppurative otitis media, adhesive otitis media or traumatic ossicular dislocation, were included in our study. We also included cases of successful tympanoplasty who suffered from mild conductive hearing loss (CHL). Patients with mixed hearing loss, otosclerosis, frozen attic syndrome or extensive cholesteatoma, were excluded. All patients 
were subjected to history taking, general examination, and otoscopic evaluation. The audiological evaluation was based on preoperative and 6 months postoperative pure tone audiometry (PTA) and air-bone gap (ABG)

\section{The operative technique}

Under general anesthesia, we used end-aural approach to elevate tympano-meatal flap. Exploration of the ossicular defect and stripping of the mucosa covering incus and stapes were done. Each patient was assigned to one of the three techniques according to length of incus defect. In the presence of incudostapedial separation or less than $30 \%$ of incus defects, $\mathrm{BC}$ was used. IT was done with more than $30 \%$ of incus defects and CBA was used in the absence of incus.

\section{IT and $C B A$}

Incus was cleaned and the short process of incus or remnant of any process was removed leaving the longer process to fashion the incus into a "teardrop" shape. Then, we used a micro drill to create a depression in the opposite side of the retained process. This depression has been made to receive stapes head. In the absence of incus, cortical bone from the mastoid region was re-shaped to the form with the above-mentioned criteria.

\section{$B C$}

The powder (polycarboxylic acid and pigments) and the liquid (water and tartaric acid) component of BC (Ketac Cem Radiopaque, ESPE; Dental Products, Seefeld, Germany) were mixed till reaching paste-like consistency then it was applied to the ossicular defect. Then we confirmed our incudo-stapedial bridging by palpating malleus and observing stapes and round window membrane movement.

\section{Statistical analysis}

All data analysis was performed using $\mathrm{R}$ software version 3.4.4 (R Foundation for Statistical Computing, Vienna, Austria ${ }^{[5]}$. Our figures were generated using the $\mathrm{R}$ package "ggplot2"[6]. Categorical outcomes were expressed as counts/frequencies. Meanwhile, the numerical outcomes were presented in the form of mean, standard deviation (SD) and standard error of the mean (SEM). Qualitative data were compared via Chi-square $(\chi 2)$ test. Pre-operative and postoperative audiometric values were compared via Wilcoxon text. Results with p-value less than 0.05 , were considered statistically significant.

\section{RESULTS}

Our study finally included 21 patients, patients with mild incus erosions less than 30\% underwent $\mathrm{BC}(33.3 \%)$, patients with incus erosions more than $30 \%$ had IT and rest of them underwent CBA due to the absence of incus (33.3\% for each technique).

Our patients ranged between 10-50 years old and out of total 21 subjects, 10 patients were male with overall $47.6 \%$ (4 patients in IT, 3 patients in CBA, and 3 patients in $\mathrm{BC}$ ) with no statistically significant difference in age, sex between the three groups (IT, CBA, and BC) with p-value (0.809, and 0.826 for each age and sex). Unilateral CHL was diagnosed in 11 patients and bilateral asymmetrical, and symmetrical CHL was diagnosed in 4, and 6 patients respectively. As well, partial malleolar erosions were found in 5 patients $(23.8 \%)$ (Table 1$)$. 
Table 1: Baseline demographics data of the included patients

\begin{tabular}{|c|c|c|c|c|c|}
\hline & Overall & Incus Transposition & Cortical bone autografting & Bone Cement & $p$ \\
\hline $\mathrm{N}$ & 21 & 7 & 7 & 7 & \\
\hline Age $(\%)$ & & & & & 0.809 \\
\hline $11-20$ & $6(28.6)$ & $2(28.6)$ & $2(28.6)$ & $2(28.6)$ & \\
\hline $21-30$ & $6(28.6)$ & $2(28.6)$ & $2(28.6)$ & $2(28.6)$ & \\
\hline $31-40$ & $6(28.6)$ & $2(28.6)$ & $1(14.3)$ & $3(42.9)$ & \\
\hline $41-50$ & $3(14.3)$ & $1(14.3)$ & $2(28.6)$ & $0(0.0)$ & \\
\hline $\operatorname{Sex}(\%)$ & & & & & 0.826 \\
\hline Male & $10(47.6)$ & $4(57.1)$ & $3(42.9)$ & $3(42.9)$ & \\
\hline Female & $11(52.4)$ & $3(42.9)$ & $4(57.1)$ & $4(57.1)$ & \\
\hline Diagnosis $(\%)$ & & & & & 0.826 \\
\hline Unilateral CHL & $11(52.4)$ & $4(57.1)$ & $3(42.9)$ & $4(57.1)$ & \\
\hline Symmetrical CHL & $6(28.6)$ & $2(28.6)$ & $2(28.6)$ & $2(28.6)$ & \\
\hline Asymmetrical CHL & $4(19)$ & $1(14.3)$ & $2(28.6)$ & $1(14.3)$ & \\
\hline Malleus (\%) & & & & & 0.159 \\
\hline Present & $16(76.2)$ & $5(71.4)$ & $4(57.1)$ & $7(100.0)$ & \\
\hline Partial erosions & $5(23.8)$ & $2(28.6)$ & $3(42.9)$ & $0(0.0)$ & \\
\hline Pre $\mathrm{ABG}^{*}($ mean $(\mathrm{SD}))$ & $44.52(10.36)$ & $33.57(5.13)$ & $35.86(5.67)$ & $26.57(5.06)$ & 0.011 \\
\hline 6mPost ABG (mean (SD)) & $27.52(6.38)$ & $15.86(1.86)$ & $19.14(2.41)$ & $10.86(6.34)$ & 0.005 \\
\hline Gain ABG (mean (SD)) & $17.00(5.50)$ & $17.71(3.40)$ & $16.71(6.68)$ & $15.71(5.85)$ & 0.795 \\
\hline Complications (\%) & & & & & 0.494 \\
\hline Transient infection & $3(14.3)$ & $1(14.3)$ & $2(28.6)$ & $0(0.0)$ & \\
\hline Transient perforation & $2(9.5)$ & $1(14.3)$ & $1(14.3)$ & $0(0.0)$ & \\
\hline Transient vertigo & $1(4.8)$ & $0(0.0)$ & $0(0.0)$ & $1(14.3)$ & \\
\hline No & $15(71.4)$ & $5(71.4)$ & $4(57.1)$ & $6(85.7)$ & \\
\hline$* \mathrm{ABG}=$ air-bone gap & & & & & \\
\hline
\end{tabular}

Audiometric and surgical outcomes: For IT group, the mean for pre- ABG was $33.57 \mathrm{~dB}$ HL with SD (5.13) $\mathrm{dB}$, after 6 months of follow-up, it became 15.86 (1.86) $\mathrm{dB}$ with mean $\mathrm{ABG}$ gain 17.71 (3.40) $\mathrm{dB}$ (Figure 1). For CBA, pre- ABG mean was 35.86 (5.67) dB HL that finally became 19.14 (2.41) dB achieving ABG gain 16.71 (6.68)

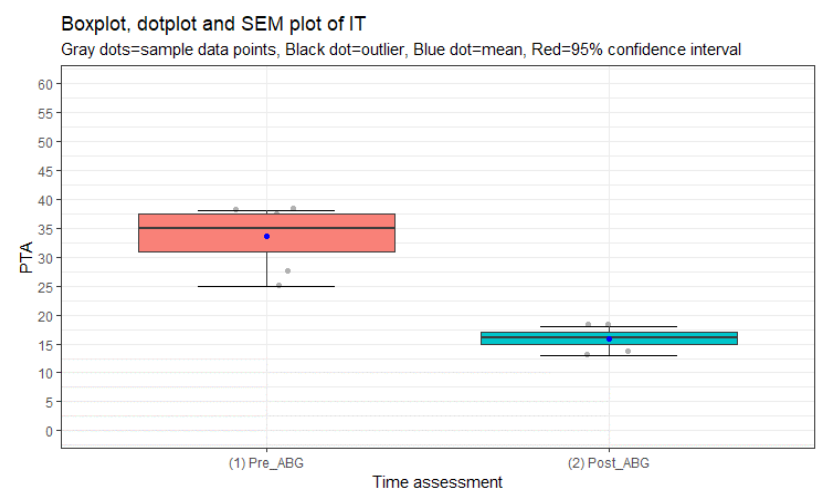

Fig. 1: Box plot, dot plot and standard error of mean (SEM) for incus transposition (IT) comparing preoperative and 6 months postoperative air bone gap (ABG).
$\mathrm{dB}$ (Figure 2). In the same context, $\mathrm{BC} \mathrm{ABG}$ was decreased from 26.57 (5.06) dB HL to 10.86 (6.34) dB with mean gain 15.71 (5.85) dB (Figure 3). No significant statistical difference between pre-post $\mathrm{ABG}$ gain in all three surgical procedures ( $p$-value $=0.80$ ).

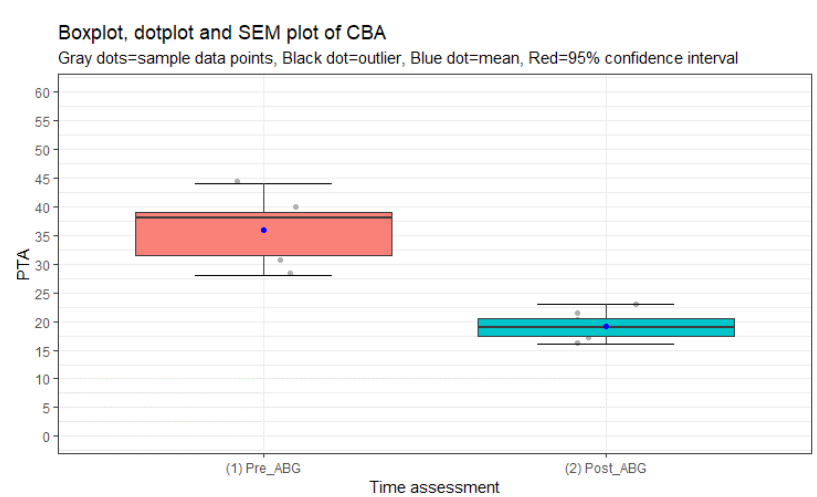

Fig. 2: Box plot, dot plot and standard error of mean (SEM) for cortical bone autografting (CBA) comparing preoperative and 6 months postoperative air bone gap (ABG). 


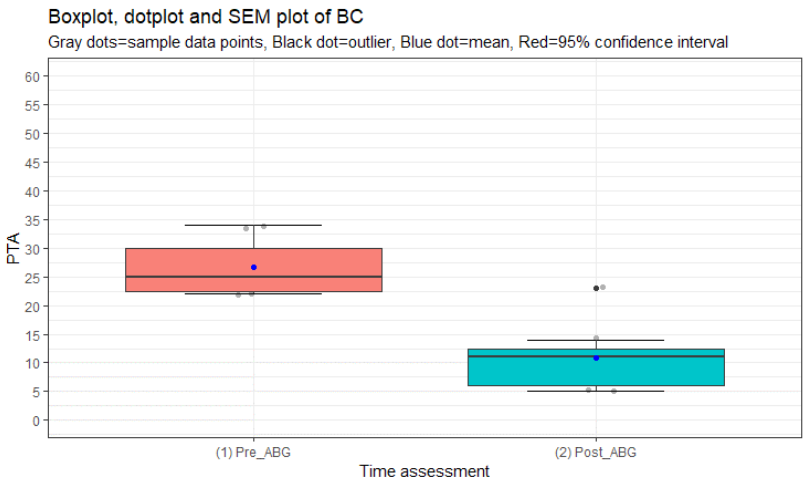

Fig. 3: Box plot, dot plot and standard error of mean (SEM) for bone cement (BC) comparing preoperative and 6 months postoperative air bone gap (ABG).

In terms of Belfast Rule of Thumb, 4 patients in each of the three technique (57.14\%), achieved less than $15 \mathrm{~dB}$ interaural difference or less than $30 \mathrm{~dB}$ hearing thresholds for PTA (Figure 4).

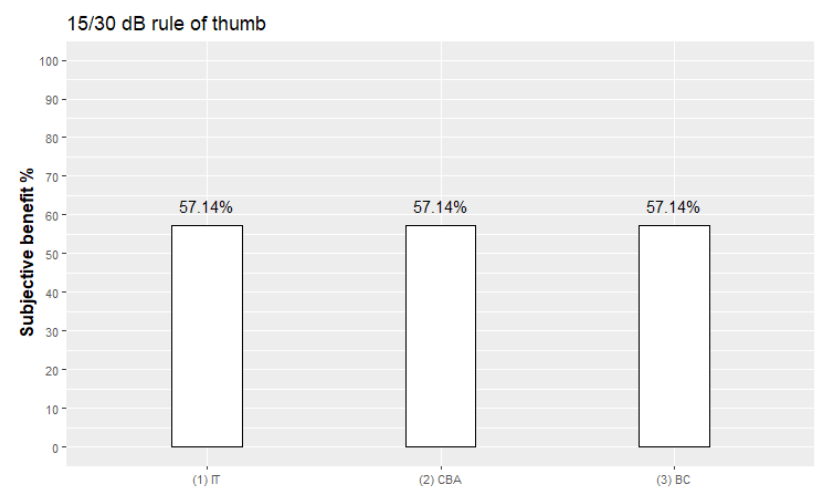

Fig. 4: Bar plot demonstrating the hearing results of the three different techniques (IT, CBA, and BC).

Two patients within the IT group had complications, one had a transient infection and the other had tympanic membrane (TM) perforation (14.3\%). For CBA group, two patients had infection $(28.6 \%)$ and one patient had transient TM perforation (14.3\%). Only one patient in the $\mathrm{BC}$ group had transient vertigo post-operatively that resolved spontaneously (14.3\%). No significant difference in adverse effects between the three groups was found $(P$-value $=0.494)$

\section{DISCUSSION}

In our study, we present the hearing results after three different techniques of ossiculoplasty in our medical center. IT, CBA, BC gave nearly similar ABG gain 17.71 (3.40), 16.71 (6.68), 15.71 (5.85) dB with no statistically significant differences among the three techniques making them as reliable techniques for cases with erosions in the long process of incus in cases with moderate hearing loss. With intact and mobile stapes in addition to other ossicular defects, two methods of tympanoplasty (namely II and III) constitute the choice for ossicular reconstruction either by a direct columellar effect of tympanic membrane or interposing graft/prosthesis between malleus handle and stapes ${ }^{[7]}$. Several materials have been proposed as a graft for ossiculoplasty ${ }^{[8-11]}$. The first autografting experience was introduced in 1960 by Hall and Rytzner ${ }^{[12]}$. Autografting, which can be done via two methods (IT, and CBA), is considered to be the most available option and takes advantage of being bio-compatible and low cost. In contrast, homograft might undergo complete resorption or carry microscopic prions disease. As well, allografting ${ }^{[13]}$, in spite of being made to be bio-compatible, is still expensive and till now, there is no solid literature agreement regarding the effectiveness of each one of allografts. Regarding the use of IT technique, Zhang et al in $2011^{[1]}$, firstly, reported that titanium prostheses added no more benefit compared to non-titanium ones and Bartel et al ${ }^{[4]}$ in 2018, secondly, concluded that IT gave comparable efficacy as PORP. We, also, here propose CBA for ossicular reconstruction in cases of moderate hearing loss. According to our findings, it gave results similar to IT and hence, can serve as the second option in the absence of incus remnants suitable for IT.

$\mathrm{BC}$ was firstly introduced from the dental field. It becomes popular nowadays with promising hearing outcomes ${ }^{[14,15,16]}$. Moreover, some argue that it can be used for lengthening of incus or even complete restoration of connection between malleus and stapes. However, results might be lower with a large gap due to incus defects. Despite the alleged neurotoxicity of $\mathrm{BC}$, we did not note this side effect in our sample and, as well, no articles reported on this issue till now ${ }^{[17]}$.

Our study summarizes the hearing results after three different techniques of ossicular reconstruction. We also provide a clear plan for ossicular reconstruction in cases of moderate hearing loss according to the complete/partial absence of incus. However, this report, still, might have several limitations as low sample size, and relatively short term of follow-up (6 months). Hence, more trials with longer follow-up periods are recommended.

\section{CONCLUSION}

This study compares the hearing results of $\mathrm{BC}$, IT, and CBA. All techniques demonstrated to be effective and reliable with good postoperative hearing results with no significant difference between all of them. IT \& CBA could be used for large incus defects and $\mathrm{BC}$ is a good alternative for closing small incudo-stapedial joint defects. The choice of the proper technique depends on the length of incus defects.

\section{CONFLICT OF INTEREST}

There are no conflict of interest.

\section{REFERENCES}

1. Zhang L-C, Zhang T-Y, Dai P, Luo J. Titanium versus non-titanium prostheses in ossiculoplasty: a metaanalysis. Acta Otolaryngol 2011; 131: 708-15 
2. Wullstein H. Theory and practice of tympanoplasty. Laryngoscope 1956; 66: 1076-93

3. Baylancicek $\mathrm{S}$, Iseri $\mathrm{M}$, Topdağ DÖ, et al. Ossicular reconstruction for incus long-process defects: bone cement or partial ossicular replacement prosthesis. Otolaryngol Head Neck Surg 2014; 151: 468-72

4. Bartel R, Cruellas F, Hamdan M, et al. Hearing results after type III tympanoplasty: incus transposition versus PORP. A systematic review. Acta Otolaryngol 2018; 138: 617-20

5. R Core Team. R: A Language and Environment for Statistical Computing. Vienna, Austria 2014; Available from: https://cran.r-project.org/bin/windows/base/ old $/ 3.4 .4 /$

6. Wickham H. ggplot2: elegant graphics for data analysis . Springer; 2016. Available from: https:// doi.org/10.1007/978-3-319-24277-4

7. Emir H, Kizilkaya Kaptan Z, Göcmen H, et al. Ossiculoplasty with intact stapes: analysis of hearing results according to the middle ear risk index. Acta Otolaryngol 2009; 129: 1088-94

8. O'Reilly RC, Cass SP, Hirsch BE, Kamerer DB, Bernat RA, Poznanovic SP. Ossiculoplasty using incus interposition: hearing results and analysis of the middle ear risk index. Otol Neurotol 2005; 26: 853-8

9. Al-Qudah M, Dawes PJD. Malleus-stapes assembly: experience with two prostheses. J Laryngol Otol 2006; 120: 736-9
10. Romanet P, Duvillard C, Delouane M. [Mastoid cortical bone grafts in ossiculoplasty]. Ann Otolaryngol Chir Cervicofac 2000; 117: 105-9

11. Kartush JM. Ossicular chain reconstruction. Capitulum to malleus. Otolaryngol Clin North Am 1994; 27 : 689-715

12. Hall A, Rytzner C. Vitality of autotransplanted ossicles. Acta Otolaryngol Suppl 1960; 158: 335-40

13. Martin AD, Harner SG. Ossicular reconstruction with titanium prosthesis. Laryngoscope 2004; 114: 61-4

14. Bora F, Yücel Z, Ceylan S, Oltulu E, Batmaz T, Avseren E. [Incudostapedial bridging ossiculoplasty with bone cement hearing results]. Kulak Burun Bogaz Ihtis Derg 20: 169-72

15. Elsheikh MN, Elsherief H, Elsherief S. Physiologic reestablishment of ossicular continuity during excision of retraction pockets: use of hydroxyapatite bone cement for rebridging the incus. Arch Otolaryngol Head Neck Surg 2006; 132: 196-9

16. Ozer E, Bayazit YA, Kanlikama M, Mumbuc S, Ozen Z. Incudostapedial rebridging ossiculoplasty with bone cement. Otol Neurotol 2002; 23: 643-6

17. Alkan S, Koşar AT, Sozen E, et al. Assessment of longand short-term neurotoxic effects of glass ionomer bone cement by electromyography and histopathologic examination: experimental study. J Otolaryngol Head Neck Surg 2008; 37: 844-50 